Rev. salud pública. 13 (6): 990-997, 2011

\title{
Prevalencia de infección por Demodex folliculorum en pacientes que acuden a consulta general de oftalmología
}

\section{The prevalence of Demodex folliculorum infection in patients attending a general ophthalmological consultation}

\author{
Virgilio Galvis-Ramírez, Alejandro Tello-Hernández, Leonardo Álvarez-Osorio y \\ Juan J. Rey-Serrano
}

Centro Oftalmológico Virgilio Galvis, Fundación Oftalmológica de Santander. Facultad de Ciencias de la Salud, Universidad Autónoma de Bucaramanga. virgiliogalvis@gmail.com, alejandrotello@gmail. com, leonardoalvarez7@hotmail.com,juanjoreys@gmail.com

Recibido 1 Mayo 2011/Enviado para Modificación 1 Diciembre 2011/Aceptado 15 Diciembre 2011

\section{RESUMEN}

Objetivos Determinar la prevalencia de infección por Demodex folliculorum en pacientes que asisten a consulta general de oftalmología, y su asociación con blefaritis.

Métodos Estudio descriptivo de prevalencia. En forma aleatoria se seleccionaron 128 sujetos que asistieron al Centro Oftalmológico Virgilio Galvis y se determinó la presencia de Demodex folliculorum en 4 pestañas de cada uno.

Resultados La prevalencia de infección por Demodex folliculorum fue 42,1\% ( $=$ $54)$. Se diagnosticó blefaritis en el $38,3 \%$ de los pacientes $(n=49)$ y el $63,2 \%$ de ellos $(n=31)$ fueron positivos para $D$. folliculorum. En el grupo sin blefaritis $(n=79)$ solo el $29,2 \%$ de los individuos tenían el ácaro $(n=23)(p=0.0003)$. Además se encontró una mayor cantidad de parásitos en los pacientes con blefaritis (índice de carga parasitaria 12,7 vs $5,1(p=0.0001)$. El $25 \%(n=32)$ de los participantes presentaban descamación en forma de cilindros y en estos el 96,9 \% tenían el ácaro $(n=31)$, mientras que en los sujetos que no tenían cilindros $(n=96)$ el ácaro estuvo presente solo en el $24 \%(n=23)$.

Conclusiones El Demodex folliculorum es un parásito que se encuentra en personas sin lesiones oculares, pero que es más frecuente y presenta una mayor carga parasitaria en pacientes con blefaritis. Nuestros resultados sugieren la necesidad de investigar la presencia de Demodex folliculorum en todo paciente con blefaritis y especialmente en los casos en que se observe presencia de descamación en forma de cilindros en las pestañas.

Palabras Clave: Blefaritis, enfermedades de los párpados, pestañas (fuente: DeCS, BIREME). 


\section{ABSTRACT}

Objectives Determining Demodex folliculorum infection prevalence in patients attending general ophthalmological consultation and such infection's association with blepharitis.

Methods This was a descriptive study of such prevalence. 128 subjects who attended the Virgilio Galvis Eye Centre were randomly selected and the presence of $D$. folliculorum was determined in 4 eyelashes taken from each of them.

Results $D$. folliculorum infection prevalence was $42.1 \%(n=54)$; blepharitis was diagnosed in $38.3 \%$ of the patients $(n=49)$ and $63.2 \%$ of them $(n=31)$ were positive for $D$. folliculorum. Only $29.2 \%$ of the individuals in the group without blepharitis $(n=79)$ had mites $(n=23)(p=0.0003)$. A higher number of parasites was found in patients with blepharitis (12.7 parasitic load index of $5.1 ; p=0.0001$ ); $25 \%(n=32)$ of the participants had scaling in the form of cylinders and $96.9 \%$ of these had the mites $(n=31)$, whereas the mite was only present in $24 \%(n=23)$ of subjects who had no cylinder-type scaling ( $n=96)$.

Conclusions Demodex folliculorum is a parasite found in people without ocular lesions; however, it is more frequent and has a higher parasite burden in patients with blepharitis. Our results suggested the need for investigating the presence of $D$. folliculorum in all patients suffering from blepharitis, especially in cases where cylinder-type scaling has been observed in their eyelashes.

Key Words: Blepharitis, diseases of the eyelids and eyelashes (source: $\mathrm{MeSH}$, $N L M)$.

$\mathrm{E}$

1 Demodex folliculorum es un parásito que reside en los folículos pilosos y glándulas sebáceas de la cara. Es un ácaro que mide en promedio 280 micras fácilmente identificable al examen directo bajo microscopía de luz (Figura 1).

Se ha relacionado la infestación por este parásito con enfermedades como rosácea, blefaritis, meibomitis, chalazión y ojo seco (1-3). Sin embargo, todavía existen controversias sobre su rol patogénico en estas enfermedades. La prevalencia de la infección por $D$. folliculorum es variable dependiendo de la población estudiada (4-10) y es muy poco conocida en nuestro medio. El objetivo del presente trabajo fue determinar la prevalencia del ácaro en pacientes que asisten a consulta general de oftalmológica y relacionarla con la presencia de blefaritis. Además proponemos un índice de carga parasitaria que se mostró útil para identificar individuos con infestación más severa por el ácaro y blefaritis. 
Figura 1. Demodex folliculorum bajo el microscopio de luz (aumento 100X)

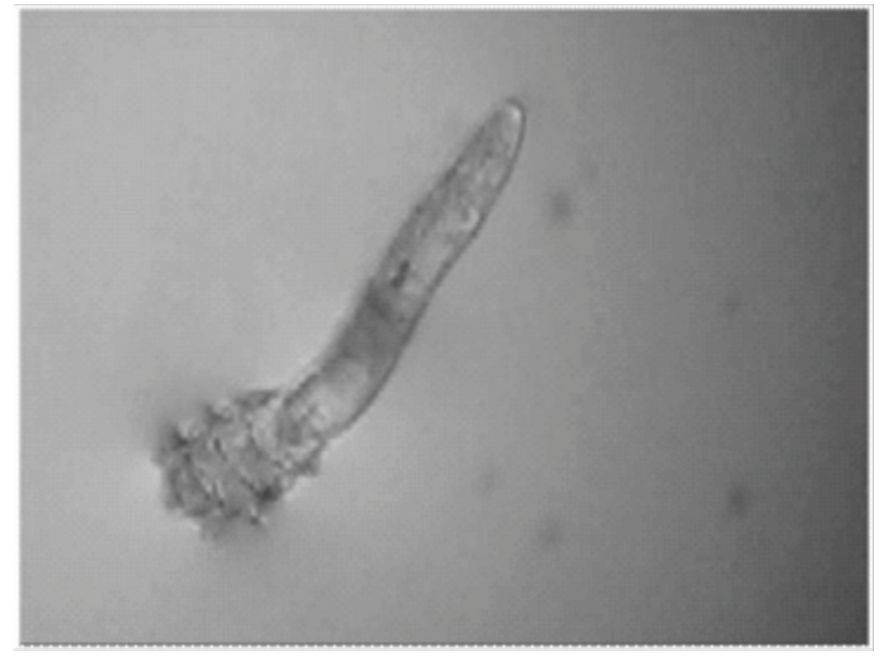

Estudio transversal de prevalencia. De los pacientes que asistieron a consulta general al Centro Oftalmológico Virgilio Galvis, se seleccionaron al azar 5 personas por 26 días (entre los meses de Marzo y Abril de 2010), a quienes se les solicitó participar en el estudio previa explicación y obtención del consentimiento informado. Se excluyeron los pacientes que hubieran recibido tratamiento para blefaritis en los últimos 6 meses, menores de 18 años, quienes hubiesen empleado cualquier sustancia para higiene palpebral y mujeres que asistían a consulta maquilladas con rímel. A cada participante se le realizó examen de los párpados con lámpara de hendidura por parte de uno de los investigadores (AT) para determinar la presencia de descamación y su tipo, colocando especial atención a la identificación de la descamación tubular en forma de cilindro en la base de las pestañas (Figura 2). Esta información se consignó en un formulario. Posteriormente, bajo lámpara de hendidura, se le retiró cuidadosamente 1 pestaña por párpado, 4 en total, seleccionándose aquellas pestañas con descamación en escamas o en forma de cilindro, en caso de haberlas. Una vez obtenidas, se colocaron sobre una lámina portaobjetos y se cubrieron con cinta adhesiva para su posterior examen bajo el microscopio de luz con el fin de determinar la presencia de Demodex folliculorum. Se consignaron los hallazgos en un formulario con las variables estudiadas. El examen al microscopio fue realizado por un sólo investigador (LA) durante las primeras 8 horas luego de haberse obtenido las pestañas. Este investigador realizó el examen en 
forma enmascarada, puesto que no conocía la historia clínica y los datos incluidos en el formulario. Se consignó en cada caso la presencia o no de $D$. folliculorum, el número de pestañas con el parásito y el número de ácaros por pestaña. Con estos resultados adicionalmente a analizar la prevalencia del D. folliculorum, diseñamos un índice de carga parasitaria compuesto por el número de pestañas infectadas y el número de ácaros totales, y otro índice que utilizó el número máximo de ácaros en una sola pestaña.

\section{RESULTADOS}

Se estudiaron un total de 128 pacientes (512 pestañas). En todos los casos que presentaban descamación en forma de cilindros se logró la conservación de los mismos en la muestra. El promedio de edad fue de 43,9 años con límites entre 18 y 86 años, 39,1 \% fueron mujeres, $49 \%$ fueron pacientes pertenecientes al régimen contributivo, $17 \%$ a los regímenes especiales y $34 \%$ particulares y con medicina prepagada.

Figura 2. Descamación en forma de cilindros en un caso de blefaritis con presencia de $D$. folliculorum

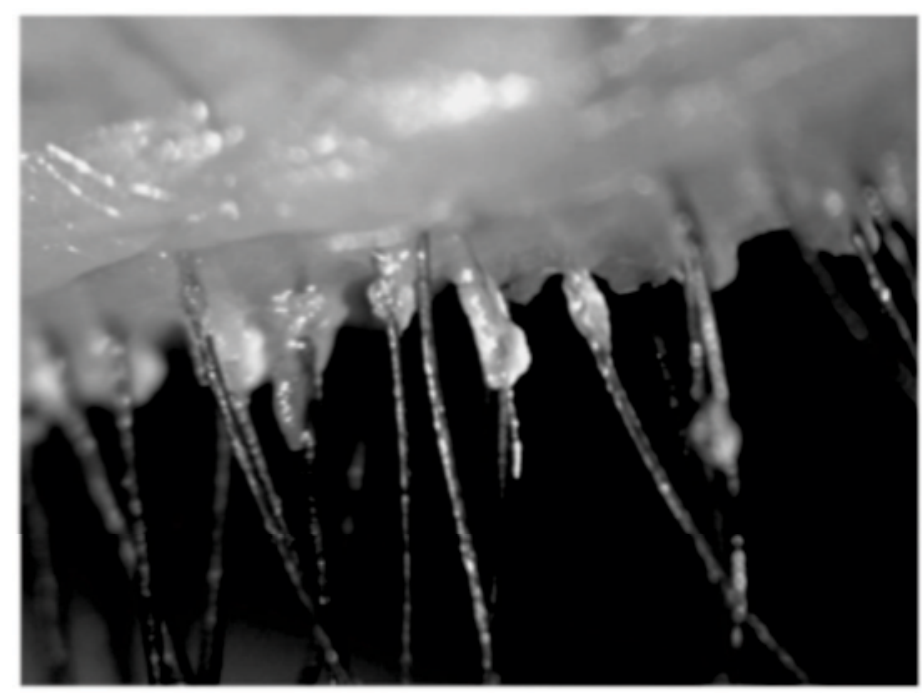

La prevalencia total de infección por Demodex fue del 42,1 \% ( $\mathrm{n}=54$. El parásito se encontró en 8 de los 41 pacientes menores de 30 años (19,5 $\%$ ), en 17 de los 35 (48,5\%) del grupo comprendido entre los 31-50 años, 
en 18 de los 36 (50\%) pacientes entre los 51-70 años y en 11 de $16(68,7$ $\%$ ) de los mayores de 71 años.

El 38,3\% (n=49) de todos los pacientes tuvieron diagnóstico de blefaritis. La presencia de Demodex folliculorum fue más frecuentemente identificado en los pacientes con blefaritis (31 de 49,63,2\%) frente a los pacientes sin blefaritis $(23$ de $79,29,2 \%)(p=0.0003)$. Además, los pacientes con blefaritis presentaron un mayor índice de carga parasitaria (número de pestañas infectadas $\mathrm{x}$ número de ácaros totales: 1,9 x 6,7=12,7) en relación a los individuos sin blefaritis $(1,6 \times 3,2=5,1)(\mathrm{p}=0.0001)$.

Treinta y dos de los individuos con blefaritis $(25 \%$ de la población total) presentaron descamación en forma de cilindros en la base de las pestañas y en estos el ácaro estuvo presente en las pestañas del 96,9\% de estos sujetos (31 pacientes). Por el contrario el parásito se encontró solo en el $24 \%$ de de los examinados que no presentaban cilindros $(n=96)$, $(\mathrm{p}=0.0001)$.

Figura 3: Izquierda: Folículo de una pestaña de un paciente con blefaritis y descamación en forma de cilindros, con 12 adultos de $D$. folliculorum adheridos a su base (aumento 100x). Derecha: Detalle a mayor aumento (400x)

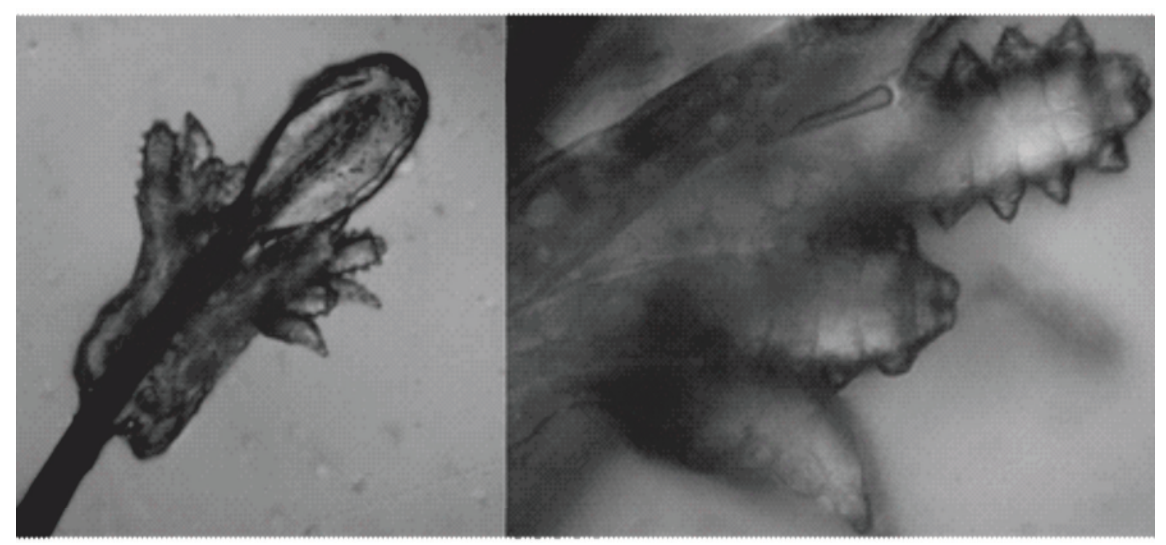

La otra medida que se utilizó fue número de ácaros en una sola pestaña. En los pacientes con cilindros y blefaritis se encontraron entre 1 y 12 ácaros por pestaña, con una cantidad de 4 ácaros o más en una pestaña en el 71,4 \% (Figura 3 ). Por el contrario en quienes se detectó el $D$. folliculorum 
pero no tenían descamación, se encontraron de 1 a 5 ácaros por pestaña, y solo un $5 \%$ de estos pacientes sin blefaritis tenían 4 ácaros o más en una pestaña. Estos resultados sugieren que clínicamente se puede presumir la presencia del parásito al evidenciar en la lámpara de hendidura unas estructuras cilíndricas en la base de las pestañas (cilindros), las cuales parecen ser prácticamente patognomónicas de su presencia.

La prevalencia del ácaro fue similar en los pacientes de diferentes regímenes de salud.

\section{DISCUSIÓN}

El Demodex folliculorum se ha encontrado en las glándulas sebáceas de la cabeza, complejos pilosebáceos y folículos pilosos de la cara, pestañas, las axilas y la región del pubis (3-4). El parásito se desplaza hacia la base de la pestaña donde se puede identificar al depilar la pestaña y observarla al microscopio de luz.

Desde hace décadas se ha discutido si este ácaro es un saprofito inofensivo o un microorganismo patógeno (5). Varios estudios en diferentes poblaciones han sido realizados intentando resolver la controversia (5-10). Así, un reporte de Dinamarca de la década de 1970 encontró al estudiar 8 pestañas tomadas por depilación de cada uno de 400 pacientes de diversos servicios de un hospital, que el $48 \%$ presentaban Demodex folliculorum. No se reportó si los pacientes tenían o no blefaritis. La prevalencia tuvo relación directa con la edad, siendo del $26 \%$ en las persona de 10 a 29 años; del $31,5 \%$ en los de 30 a 49 años; del $53 \%$ en los de edades entre 50 y 69 años; y del $72,3 \%$ en mayores de 71 años (5). Posteriormente, en una serie de 568 pacientes con blefaritis en Polonia se encontró una prevalencia de Demodex folliculorum del 68 \%; sin embargo en este estudio no se reportó la prevalencia del ácaro en la población sin blefaritis (6). En Bélgica se encontró un $25 \%$ de prevalencia en sujetos normales y un $51 \%$ en pacientes con blefaritis (7). En Turquía, entre los 48 pacientes sanos estudiados con edades entre 16 y 81 años, la prevalencia fue de $4,16 \%$, mientras que entre 37 pacientes con blefaritis fue de $29,7 \%$, diferencia estadísticamente significativa. En este trabajo no se reportó el número de pestañas que se examinaron en cada paciente (8). En España encontraron que la incidencia de Demodex folliculorum en el 
grupo control (105 pacientes) fue $18 \%$ con un promedio de 0,08 ácaros/ pestaña mientras que en el $75 \%$ de los pacientes con blefaritis crónica (20 pacientes) se encontró el Demodex folliculorum, con un promedio de 0,69 ácaros/pestaña, diferencia estadísticamente significativa $(\mathrm{p}=0,006)$ (9). Estos estudios realizados con un número limitado de pacientes son sugestivos de que la presencia del ácaro se asocia a un mayor riesgo de presentar blefaritis. Sin embargo el estudio mas grande y mejor diseñado, realizado en Turquía, que incluyó 170 pacientes con blefaritis seborreica y 330 normales, en los cuales se examinaron 12 pestañas de cada paciente, no mostró diferencias significativas en la prevalencia de $D$. folliculorum (28,8\% en los pacientes con blefaritis y $26,7 \%$ en los controles) ni diferencias significativas entre los grupos de edad (10). Las razones de estas discrepancias no han sido definidas por lo que es necesario la realización de más estudios epidemiológicos que clarifiquen la controversia. En nuestro estudio realizado en una muestra tomada al azar de entre quienes asistieron a consulta oftalmológica, aunque encontramos que el $D$. folliculorum es un parásito que se puede encontrar en individuos aparentemente sanos, pudiendo habitar en las pestañas sin ocasionar sintomatología, como ya otros investigadores lo han reportado, confirma la reportada asociación entre la presencia de Demodex folliculorum y blefaritis (5-9). Encontramos que la prevalencia del ácaro es mayor en pacientes de mayor edad y en pacientes con blefaritis-específicamente cuando esta se acompaña de descamación en forma de cilindros en la base de las pestañas.

La técnica utilizada para la toma de las pestañas seleccionando aquellas que presentan cilindros tiene la ventaja de ser sencilla, permite extraer una menor cantidad de pestañas con menor incomodidad para el paciente y permite la toma directa por parte del oftalmólogo, mejorando la calidad al poder seleccionar las pestañas más apropiadas bajo lámpara de hendidura. El índice que utilizamos con base en el número de pestañas afectadas multiplicada por el número de ácaros totales parece ser una alternativa fácil para medir la carga parasitaria. Tanto índice de carga parasitaria y como el número máximo de ácaros en una pestaña pueden ser útiles para el diagnóstico.

En conclusión, los resultados del presente estudio sugieren la necesidad de investigar la presencia de Demodex foliculorum en todo paciente con blefaritis y especialmente ante la presencia de descamación en forma de cilindros al examen oftalmológico 
Conflictos de interés: Ninguno

\section{REFERENCIAS}

1. Galvis V, Tello A, Álvarez L. Blefaritis por Demodex. Review of ophthalmology en español 2010;38:30-32.

2. Forton F, Seys B. Density of Demodex folliculorum in rosacea: a case control study using standardized skin surface biopsy. British J Dermatol 1993;128:650-59.

3. Hoekzema R, Hulsebosch HJ, Bos JD. Demodicidosis or rosacea: What did we treat? $\mathrm{Br} \mathrm{J}$ Dermatol. 1995;133(2):294-99.

4. Gelvez RM, Rodríguez R. Blefaritis acárica por Demodex folliculorum. Hallazgos biomicroscópicos. Arch Soc Españ Oftalmol. 1993; 65:455-62.7.

5. Norn MS. Demodex folliculorum. Incidence and possible pathogenic role in the human eyelid: Capitulo IV: Incidence in the ocular region of a clinical material, Demodex folliculorum. Acta Ophthalmol Suppl. 1970;108: 43-52.

6. Humiczewska M. [Demodex folliculorum and Demodex brevis (Acarida) as the factors of chronic marginal blepharitis].Wiad Parazytol. 1991;37(1):127-30.

7. Uyttebroeck W, Nijs I, Maudgal PC, Missotten L. Incidence of Demodex folliculorum on the eyelash follicle in normal people and in blepharitis patients. Bull Soc Belge Ophtalmol. 1982;201:83-7.

8. Türk M, Oztürk I, Sener AG, Küçükbay S, Afşar I, Maden A. Comparison of incidence of Demodex folliculorum on the eyelash follicule in normal people and blepharitis patients. Turkiye Parazitol Derg. 2007;31(4):296-7.

9. Rodríguez AE, Ferrer C, Alió JL. Chronic blepharitis and Demodex. Arch Soc Esp Oftalmol, 80(11):635-42. 2.005.

10. Kemal M, Sümer Z, Toker MI, Erdoğan H, Topalkara A, Akbulut M. Ophthalmic Epidemiol. 2005 Aug;12(4):287-90. The Prevalence of Demodex folliculorum in blepharitis patients and the normal population. 